\title{
HYERS-ULAM STABILITY OF FIRST ORDER LINEAR DIFFERENCE OPERATORS ON BANACH SPACE
}

\author{
A. K. Tripathy* and P. Senapati ${ }^{\dagger}$ \\ Department of Mathematics \\ Sambalpur University \\ Sambalpur-768019, INDIA
}

\begin{abstract}
In this work, the Hyers-Ulam stability of first order linear difference operator $T_{p}$ defined by

$$
\left(T_{p} u\right)(n)=\triangle u(n)-p(n) u(n)
$$

is studied on the Banach space $X=l_{\infty}$, where $p(n)$ is a sequence of reals.

Keywords : Hyers-Ulam stability, difference equation.

Mathematics Subject Classification (2010) : 39A45, 39B42.
\end{abstract}

\section{Introduction}

Let $X=l_{\infty}$ be the Banach space of all real valued functions $u(n)$ defined for $n \geq 0$. Let $D(I, X)$ be the linear space of all $X$-valued functions on an open interval $I=(a, b+1) \subset$ $\mathbb{N}(0)=\{0,1,2, \ldots\}, a<b$. We define

$$
\|f\|_{\infty}=\sup \{\|f(n)\|, n \in I\}
$$

for every $f \in D(I, X)$. Define the linear difference operator $T_{p}: D(I, X) \rightarrow D(I, X)$ by

$$
\left(T_{p} u\right)(n)=\triangle u(n)-p(n) u(n), \forall u \in D(I, X), \forall n \in I .
$$

\footnotetext{
*Corresponding Author ; Email : arun_tripathy70@rediffmail.com

†Email: prangyasenapati2012@gmail.com
} 
We notice that $T_{p}$ is onto. Indeed, for every $v \in D(I, X)$

$$
u(n)=\prod_{i=n_{0}}^{n-1}(1+p(i)) \sum_{s=n_{0}}^{n-1} v(s)\left(\prod_{i=n_{0}}^{s}(1+p(i))\right)^{-1}
$$

satisfies $T_{p}=v$. Conversely, the general solution of $T_{p}=v$ is of the form

$$
u(n)=\prod_{i=n_{0}}^{n-1}(1+p(i))\left[x_{0}+\sum_{s=n_{0}}^{n-1} v(s)\left(\prod_{i=n_{0}}^{s}(1+p(i))\right)^{-1}\right]
$$

for every $n_{0}, n \in I$ and $x_{0} \in X$ is an arbitrary element.

DEFINITION 1.1 We say that the difference operator $T_{p}$ has the Hyers-Ulam stability, if there exists a constant $K \geq 0$ with the property: For every $\epsilon \geq 0$ and $u, v \in D(I, X)$ satisfying $\left\|T_{p} u-v\right\|_{\infty} \leq \epsilon$ there exists $u_{0} \in D(I, X)$ such that $T_{p} u_{0}=v$ and $\left\|u-u_{o}\right\|_{\infty} \leq K \epsilon$. We call such $K$ a HUS constant for $T_{p}$. If, in addition, minimum of all such $K^{\prime} s$ exists, then we call it the HUS constant for $T_{p}$.

In 1940 [25], S. M. Ulam posed the problem: When can we assert that approximate solution of a functional equation can be approximated by a solution of the corresponding equation? before the audience at the University of Wisconsin which was first answered by D. H. Hyers [5] on Banach space. Thereafter, T. Aoki [2], D. H. Bourgin [3] and Th. M. Rassias [20] improved the result of Hyers. For more details, we refer the readers to the books by Hyers et al. [6] and monograph by S. M. Jung [11]. After that many researchers have extended the Ulam's stability problems to other functional equations and generalized Hyer's result in various directions. Recently, the Ulam's stability problem for functional equations has been replaced by stability of differential and difference equations (see for e.g. [[1], [7], [8]- [10], [12], [13], [14], [15], [18], [21], [23]], [19], [24]).

In [24], Tripathy has studied the Hyers-Ulam stability of the following difference equations:

$$
\begin{aligned}
& y(n+1)-p(n) y(n)-r(n)=0, \\
& y(n+2)+\alpha y(n+1)+\beta y(n)=0, \\
& y(n+2)+\alpha y(n+1)+\beta y(n)=r(n), \\
& y(n+2)-\alpha(n) y(n+1)+\beta(n) y(n)=r(n),
\end{aligned}
$$

where $\alpha, \beta, p$ and $r$ are sequences of reals. In this work, our objective is to study the HyersUlam stability of the operator $T_{p}$ followed by (1.1) on Banach space in accordance with the necessary and sufficient condition.

On Hyers-Ulam stability, several works have been done in the direction of differential equations. Obloza seems to be the first author who has investigated the Hyers-Ulam stability of linear differential equations(see for e.g[[18], [21]] ). After that, Alsina and Ger published 
their work [1], where they have proved the Hyers-Ulam stability of the differential equation $y^{\prime}(t)=y(t)$. We remark here that absolutely there is no such work on (1.1). We use the following lemma in our next discussion:

LEMMA 1.2 [22] Let $C$ be a symmetric set, that is, $C=-C$ in a Banach space $B$. For each $y \in B$, we have

$$
\sup _{x \in C}\|y+x\| \geq \sup _{x \in C}\|x\|
$$

\section{Hyers-Ulam Stability Results}

In this section, we discuss the necessary and sufficient conditions for Hyers-Ulam stability of the operator $T_{p}$ followed by (1.1) on the Banach space $X=l_{\infty}$. We use the following notions for our use in the sequel:

$$
\begin{aligned}
& 1+p(n) \neq 0, \quad P(n)=\left(\prod_{i=0}^{n-1}(1+p(i))\right)^{-1}, n \in \mathbb{N}(0), \\
& \alpha_{p}=\sup _{n \geq 0} \frac{1}{|P(n)|} \sum_{m=n}^{\infty}|P(m+1)|, \quad \beta_{p}=\sup _{n \geq 0} \frac{1}{|P(n)|} \sum_{m=0}^{n-1}|P(m+1)| .
\end{aligned}
$$

We use the sign convention $\left(\prod_{i=0}^{n-1}(1+p(i))\right)=1$ for $n-1<0$.

THEOREM 2.1 Let $T_{p}: D(\mathbb{N}(0), X) \rightarrow D(\mathbb{N}(0), X)$ be the linear operator defined by

$$
\left(T_{p} u\right)(n)=\triangle u(n)-p(n) u(n), \forall u \in D(\mathbb{N}(0), X), \forall n \in \mathbb{N}(0) .
$$

If $\inf _{n \geq 0}|P(n)|=0$, then $T_{p}$ has the Hyers-Ulam stability with HUS constant $\alpha_{p}$ if and only if $\alpha_{p}<\infty$.

Proof. Let $\epsilon \geq 0$ and $u, v \in D(\mathbb{N}(0), X)$ satisfy $\left\|T_{p} u-v\right\|_{\infty} \leq \epsilon$. Set $w=T_{p} u-v$. Then $\|w\|_{\infty} \leq \epsilon$ and $T_{p} u=v+w$ implies that

$$
\begin{aligned}
u(n) & =\prod_{i=0}^{n-1}(1+p(i))\left[u(0)+\sum_{s=0}^{n-1}(v+w)\left(\prod_{i=0}^{s}(1+p(i))\right)^{-1}\right] \\
& =\prod_{i=0}^{n-1}(1+p(i)) \sum_{s=0}^{n-1} v(s)\left(\prod_{i=0}^{s}(1+p(i))\right)^{-1} \\
& +\prod_{i=0}^{n-1}(1+p(i))\left[u(0)+\sum_{s=0}^{n-1} w(s)\left(\prod_{i=0}^{s}(1+p(i))\right)^{-1}\right] \\
& =\frac{1}{P(n)} \sum_{s=0}^{n-1} v(s) P(s+1)+\frac{1}{P(n)}\left[u(0)+\sum_{s=0}^{n-1} w(s) P(s+1)\right]
\end{aligned}
$$


and $\sum_{s=0}^{n-1} w(s) P(s+1) \in X$ exists for every $n \in \mathbb{N}(0)$.

Now, we consider the case when $\alpha_{p}<\infty$. For each $n \in \mathbb{N}(0)$, it is easy to see that $u(n)=\frac{1}{P(n)} \sum_{s=0}^{n-1} v(s) P(s+1)+\frac{1}{P(n)}\left[u(0)+\sum_{s=0}^{\infty} w(s) P(s+1)\right]-\frac{1}{P(n)} \sum_{s=n}^{\infty} w(s) P(s+1)$.

If we put $x_{0}=u(0)+\sum_{s=0}^{\infty} w(s) P(s+1)$ and

$$
u_{0}(n)=\frac{1}{P(n)}\left[x_{0}+\sum_{s=0}^{n-1} v(s) P(s+1)\right]
$$

then $T_{p} u_{0}=v$ and

$$
u(n)=u_{0}(n)-\frac{1}{P(n)} \sum_{s=n}^{\infty} w(s) P(s+1) .
$$

Therefore,

$$
\left\|u(n)-u_{0}(n)\right\|=\frac{1}{|P(n)|}\left\|\sum_{s=n}^{\infty} w(s) P(s+1)\right\|
$$

implies that

$$
\left\|u-u_{0}\right\|_{\infty} \leq \sup _{n \geq 0} \frac{\epsilon}{|P(n)|} \sum_{s=n}^{\infty}|P(s+1)| \leq \epsilon \alpha_{p} .
$$

Hence, $T_{p}$ has the Hyers-Ulam stability with HUS constant $\alpha_{p}$. We claim that $u_{0}$ is determined uniquely. If not, let $u_{1}, u_{2} \in D(\mathbb{N}(0), X)$ be such that

$$
T_{p} u_{i}=v \text { and }\left\|u-u_{i}\right\|_{\infty} \leq M_{i}<\infty, \quad(i=1,2) .
$$

Hence for $T_{p} u_{i}=v$, we can find $x_{i}$ for $i=1,2$ such that

$$
u_{i}(n)=\frac{1}{P(n)}\left[x_{i}+\sum_{s=0}^{n-1} v(s) P(s+1)\right], \forall n \in \mathbb{N}(0) .
$$

Therefore, it follows that

$$
\begin{aligned}
\left\|x_{1}-x_{2}\right\|=|P(n)|\left\|u_{1}(n)-u_{2}(n)\right\| & \leq|P(n)|\left\|u_{1}-u_{2}\right\|_{\infty} \\
& \leq|P(n)|\left(M_{1}+M_{2}\right), \forall n \in \mathbb{N}(0),
\end{aligned}
$$

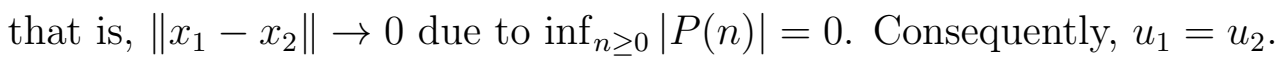

Conversely, let's fix $x_{0} \in X$ such that $\left\|x_{0}\right\|=1$. Set $v(n)=\frac{|P(n+1)|}{P(n+1)} x_{0}$ for every $n \in \mathbb{N}(0)$. Then for $v \in D(\mathbb{N}(0), X)$ we can find $u \in D(\mathbb{N}(0), X)$ such that

$$
u(n)=\frac{1}{P(n)} \sum_{s=0}^{n-1} v(s) P(s+1)=\frac{1}{P(n)} \sum_{s=0}^{n-1}|P(s+1)| x_{0}
$$


for which $T_{p} u=v$ and hence $\left\|T_{p} u\right\|_{\infty}=\|v\|_{\infty}=1$. Let $K$ be an arbitrary HUS constant for $T_{p}$. So, we can find $u^{*} \in D(\mathbb{N}(0), X)$ such that

$$
T_{p} u^{*}=0 \text { and }\left\|u-u^{*}\right\|_{\infty} \leq K .
$$

It is easy to verify that $u^{*}=\frac{x_{1}}{P(n)}$ for every $n \in \mathbb{N}(0)$, where $x_{1}=u^{*}(0) \in X$. Therefore,

$$
\left\|\sum_{s=0}^{n-1}|P(s+1)| x_{0}-x_{1}\right\|=|P(n)||| \frac{1}{P(n)} \sum_{s=0}^{n-1}|P(s+1)| x_{0}-u^{*} \| \leq K|P(n)|, n \in \mathbb{N}(0)
$$

As $\inf _{n \geq 0}|P(n)|=0$, we can find a strictly monotonic increasing set of values $\left\{n_{j}\right\}_{j \in \mathbb{N}} \subset \mathbb{N}(0)$ such that

$$
n_{j} \rightarrow \infty \text { as } j \rightarrow \infty \text { and }\left|P\left(n_{j}\right)\right|<\frac{1}{j}, j \in \mathbb{N} \text {. }
$$

From (2.3) it follows that

$$
\left|\left\|\sum_{s=0}^{n_{j}-1}|P(s+1)| x_{0}\right\|-\left\|x_{1}\right\|\right| \leq \frac{K}{j}
$$

that is,

$$
\sum_{s=0}^{n_{j}-1}|P(s+1)|<\infty \text { as } j \rightarrow \infty
$$

Also, from (2.2) it is immediate that $\sum_{s=0}^{\infty}|P(s+1)| x_{0}=x_{1}$. Consequently,

$\sum_{s=n}^{\infty}|P(s+1)|=\left\|\sum_{s=0}^{n-1}|P(s+1)| x_{0}-x_{1}\right\|=\left\|\sum_{s=0}^{n-1}|P(s+1)| x_{0}-\sum_{s=0}^{\infty}|P(s+1)| x_{0}\right\| \leq K|P(n)|$

implies that $\alpha_{p} \leq K<\infty$. Since $K$ is an arbitrary HUS constant, then $\alpha_{p}$ itself is the HUS constant for $T_{p}$. This completes the proof of the theorem.

REMARK 2.2 We predict that $\beta_{p}$ could be infinity when $\alpha_{p}$ is finite. In this case, $\sum|P(m)|<\infty$. Ultimately, $\inf _{n \geq 0}|P(n)|=0$. So, we can find a strictly monotonic increasing set of values $\left\{n_{j}\right\}_{j \in \mathbb{N}} \subset \mathbb{N}(0)$ such that

$$
n_{j} \rightarrow \infty \text { as } j \rightarrow \infty \text { and }\left|P\left(n_{j}\right)\right|<\frac{1}{j}, j \in \mathbb{N} .
$$

Consequently,

$$
\beta_{p} \geq \frac{1}{\left|P\left(n_{j}\right)\right|} \sum_{m=0}^{n_{j}-1}|P(m+1)|>j \sum_{m=0}^{n_{j}-1}|P(m+1)| \rightarrow \infty \text { as } j \rightarrow \infty .
$$


THEOREM 2.3 Let $T_{p}: D(\mathbb{N}(0), X) \rightarrow D(\mathbb{N}(0), X)$ be the linear operator defined by (2.1). If $\inf _{n \geq 0}|P(n)|>0$, then $T_{p}$ has the Hyers-Ulam stability with HUS constant $\beta_{p}$ if and only if $\beta_{p}<\infty$.

Proof. We proceed as in the proof of Theorem 2.1 to obtain (2.2). If we denote

$$
u_{3}(n)=\frac{1}{P(n)}\left[u(0)+\sum_{s=0}^{n-1} v(s) P(s+1)\right]
$$

then $T_{p} u_{3}=v$ and

$$
u(n)=u_{3}(n)+\frac{1}{P(n)} \sum_{s=0}^{n-1} w(s) P(s+1)
$$

implies that

$$
\left\|u-u_{3}\right\|_{\infty} \leq \sup _{n \geq 0} \frac{\epsilon}{|P(n)|} \sum_{s=0}^{n-1}|P(s+1)| \leq \epsilon \beta_{p} .
$$

Hence, $T_{p}$ has the Hyers-Ulam stability with HUS constant $\beta_{p}$.

Assume that $\inf _{n \geq 0}|P(n)|>0$. Proceeding as in the converse part of Theorem 2.1, we have $u^{*}(n)=\frac{x_{1}}{P(n)}$ for $n \in \mathbb{N}(0)$. Hence,

$$
\sup _{n \geq 0}\left\|u_{0}(n)\right\| \leq \frac{\left\|x_{1}\right\|}{\inf _{n \geq 0}|P(n)|}<\infty
$$

implies that $\left\|x_{1}\right\| \leq\left\|u_{0}\right\|_{\infty}|P(n)|$ for every $n \in \mathbb{N}(0)$. Therefore,

$$
\sum_{m=0}^{n-1}|P(m+1)|=\left\|\sum_{m=0}^{n-1}|P(m+1)| x_{0}\right\| \leq\left(K+\left\|u_{0}\right\|_{\infty}\right)|P(n)|,
$$

that is, $\beta_{p} \leq\left(K+\left\|u_{0}\right\|_{\infty}\right)<\infty$. Since $K$ is an arbitrary HUS constant, then $\beta_{p}$ is the HUS constant for $T_{p}$. Hence, the theorem is proved.

REMARK 2.4 In Theorem 2.1, we have seen that the uniqueness is true when $\alpha_{p}<\infty$. However, the same may not be true for the case when $\beta_{p}<\infty$. In other words, if $K$ is an arbitrary constant with $\beta_{p}<K$, then for every $\epsilon>0$ and $u, v \in D(\mathbb{N}(0), X)$ satisfying $\left\|T_{p} u-v\right\|_{\infty} \leq \epsilon$, we can find infinitely many $w \in D(\mathbb{N}(0), X)$ such that $T_{p} w=v$ and $\|u-w\|_{\infty} \leq K \epsilon$.

Indeed, $T_{p}$ has the Hyers-Ulam stability due to Theorem 2.2 and (2.4) holds. Due to Theorem 2.4, let's put $\sigma=\inf _{n \geq 0}|P(n)|$. For each $x \in X$ with $\left\|x-u_{3}\right\|_{\infty} \leq \sigma \epsilon\left(K-\beta_{p}\right)$, we can define $u_{x} \in D(\mathbb{N}(0), X)$ by

$$
u_{x}(n)=\frac{1}{P(n)}\left[x+\sum_{s=0}^{n-1} v(s) P(s+1)\right]
$$


such that $T_{p} u_{x}=v$ and

$$
\begin{aligned}
\left\|u(n)-u_{x}(n)\right\| & \leq\left\|u(n)-u_{3}(n)\right\|+\left\|u_{3}(n)-u_{x}(n)\right\| \\
& \leq \epsilon \beta_{p}+\frac{1}{|P(n)|}\left\|x-u_{3}(n)\right\| \\
& \leq \epsilon \beta_{p}+\frac{\sigma \epsilon}{|P(n)|}\left(K-\beta_{p}\right) \leq K \epsilon
\end{aligned}
$$

for every $n \in \mathbb{N}(0)$. Hence, continuing in this way we can find many $w \in D(\mathbb{N}(0), X)$ such that $T_{p} w=v$ and $\|u-w\|_{\infty} \leq K \epsilon$.

REMARK 2.5 Since the uniqueness doesn't hold in case when $\beta_{p}<\infty$, then the simultaneous question is whether the infimum of all HUS constants, that is,

$$
\inf _{x \in X} \sup _{\substack{w \in D(\mathbb{N}(0), X) \\\|w\|_{\infty} \leq 1}} \sup _{n \geq 0}\left\|\frac{1}{P(n)}\left[x+\sum_{s=0}^{n-1} w(s) P(s+1)\right]\right\|
$$

if it exists for $T_{p}$ is a HUS constant or not. Indeed, if we denote

$$
L_{T_{p}}=\inf _{x \in X} \sup _{\substack{w \in D(\mathbb{N}(0), X) \\\|w\|_{\infty} \leq 1}} \sup _{n \geq 0}\left\|\frac{1}{P(n)}\left[x+\sum_{s=0}^{n-1} w(s) P(s+1)\right]\right\|
$$

and

$$
L_{0}(x)=\sup _{\substack{w \in D(\mathbb{N}(0), X) \\\|w\|_{\infty} \leq 1}} \sup _{n \geq 0}\left\|\frac{1}{P(n)}\left[x+\sum_{s=0}^{n-1} w(s) P(s+1)\right]\right\|,
$$

then it is enough to verify that $L_{T_{p}}=\inf _{x \in X} L_{0}(x)$.

If $L_{T_{p}}=\infty$, then there is nothing to verify. Assume that $L_{T_{p}}<\infty$. Let $K$ be an arbitrary HUS constant for $T_{p}$. Then for any $w \in D(\mathbb{N}(0), X)$ with $\|w\|_{\infty} \leq 1$, there exists $u_{0} \in D(\mathbb{N}(0), X)$ such that $T_{p} u_{0}=w$ and $\left\|u_{0}\right\|_{\infty} \leq K$ with

$$
u_{0}(n)=\frac{1}{P(n)}\left[x_{0}+\sum_{s=0}^{n-1} w(s) P(s+1)\right]
$$

for some $x_{0} \in X$. Indeed, $L_{0}(x) \leq K$. Since $K$ is an arbitrary HUS constant for $T_{p}$, then it follows that $L_{T_{p}} \geq \inf _{x \in X} L_{0}(x)$. Conversely, we show that $L_{T_{p}} \leq \inf _{x \in X} L_{0}(x)$. We may assume that $\inf _{x \in X} L_{0}(x)<\infty$.

Here, we assert that $L_{0}(x)$ is a HUS constant for $T_{p}$, that is, for any $\epsilon>0$ and $u, v \in$ $D(\mathbb{N}(0), X)$ with $\left\|T_{p} u-v\right\| \leq \epsilon$, there exists $u_{0} \in D(\mathbb{N}(0), X)$ such that $T_{p} u_{0}=v$ and $\left\|u-u_{0}\right\|_{\infty} \leq \epsilon L_{0}(x)$. 
If we put $\epsilon w=T_{p} u-v$ for $u, v \in D(\mathbb{N}(0), X)$, then $\|w\|_{\infty} \leq 1$. Hence for $\epsilon w+v=T_{p} u$ and for any arbitrary $x_{1} \in X$, we have

$$
u(n)=\frac{1}{P(n)}\left[x_{1}+\epsilon \sum_{s=0}^{n-1} w(s) P(s+1)+\sum_{s=0}^{n-1} v(s) P(s+1)\right]
$$

for any $n \in \mathbb{N}(0)$. Let

$$
u_{0}(n)=\frac{1}{P(n)}\left[x_{1}-\epsilon x+\sum_{s=0}^{n-1} v(s) P(s+1)\right], n \in \mathbb{N}(0) .
$$

Then $u_{0} \in D(\mathbb{N}(0), X)$ and $T_{p} u_{0}=v$. Consequently,

$$
\begin{aligned}
\left\|u-u_{0}\right\|_{\infty} & =\sup _{n \geq 0}\left\|u(n)-u_{0}(n)\right\| \\
& =\sup _{n \geq 0}\left\|\frac{\epsilon}{P(n)}\left[x+\sum_{s=0}^{n-1} w(s) P(s+1)\right]\right\| \leq \epsilon L_{0}(x)
\end{aligned}
$$

due to the fact that $\|w\|_{\infty} \leq 1$. Hence, $L_{0}(x)$ is a HUS constant for $T_{p}$. Thus, $L_{T_{p}} \leq L_{0}(x)$. Since $x \in X$ is arbitrary, then it follows that $L_{T_{p}} \leq \inf _{x \in X} L_{0}(x)$.

THEOREM 2.6 Let $T_{p}: D(\mathbb{N}(0), X) \rightarrow D(\mathbb{N}(0), X)$ be the linear operator defined by (2.1). If $\beta_{p}<\infty$, then $T_{p}$ has the Hyers-Ulam stability with HUS constant $L_{T_{p}}$.

Proof. Suppose that $\beta_{p}<\infty$. Then by Theorem 2.2, $T_{p}$ has the Hyers-Ulam stability with HUS constant $\beta_{p}$. Because the uniqueness doesn't hold in case when $\beta_{p}<\infty$ due to Remark 2.4 and $L_{T_{p}}<\infty$ exists due to Remark 2.5, then it is sufficient to show that $L_{T_{p}}=\beta_{p}$. By definition, $L_{T_{p}} \leq \beta_{p}$. Hence, we need to show that $L_{T_{p}} \geq \beta_{p}$ only. Define a linear operator $S: D(\mathbb{N}(0), X) \rightarrow D(\mathbb{N}(0), X)$ by

$$
(S w)(n)=\frac{1}{P(n)} \sum_{s=0}^{n-1} w(s) P(s+1), \quad \forall n \in \mathbb{N}(0), \quad w \in D(\mathbb{N}(0), X) .
$$

Then for all $w \in D(\mathbb{N}(0), X)$,

$$
\begin{aligned}
\|S w\|_{\infty}=\sup _{n \geq 0}\|(S w)(n)\| & =\sup _{n \geq 0}\left\|\frac{1}{P(n)} \sum_{s=0}^{n-1} w(s) P(s+1)\right\| \\
& \leq \sup _{n \geq 0} \frac{\|w\|_{\infty}}{|P(n)|} \sum_{s=0}^{n-1}|P(s+1)|=\beta_{p}\|w\|_{\infty}<\infty .
\end{aligned}
$$

Hence, $S$ is a bounded linear operator with $\|S\| \leq \beta_{p}$. Moreover, if $x_{0}$ is a unit element of $X$ and $u_{0}=\frac{|P(n)|}{P(n)} x_{0}$ for $n \in \mathbb{N}(0)$, then $u_{0} \in D(\mathbb{N}(0), X)$ and $\left\|u_{0}\right\|_{\infty}=1$. Consequently, $\left\|S u_{0}\right\|_{\infty}=\beta_{p}$ and hence $\|S\| \geq \beta_{p}$. Therefore, $\|S\|=\beta_{p}$. 
Since $|P(0)|=1$, then we can find $n_{1}>0$ such that $|P(n)| \geq \frac{1}{2}$ for $n \geq n_{1}+1$. Thus,

$$
\beta_{p} \geq \frac{1}{|P(n)|} \sum_{s=0}^{n-1}|P(s+1)| \geq \frac{1}{|P(n)|} \sum_{s=n_{1}}^{n-1}|P(s+1)| \geq \frac{\left(n-n_{1}\right)}{2|P(n)|},
$$

that is, $\frac{1}{|P(n)|} \leq 2 \beta_{p}<\infty$. Therefore, if we choose $x \in X$ arbitrary, then it follows that $\frac{x}{|P(n)|} \in D(\mathbb{N}(0), X)$. We notice that the set $\left(\left\{w \in D(\mathbb{N}(0), X):\|w\|_{\infty} \leq 1\right\}\right)$ is a symmetric set of $D(\mathbb{N}(0), X)$. Applying Lemma 1.2, we obtain

$$
\begin{gathered}
\sup _{\substack{w \in D(\mathbb{N}(0), X) \\
\|w\|_{\infty} \leq 1}} \sup _{n \geq 0}\left\|\frac{1}{P(n)}\left[x+\sum_{s=0}^{n-1} w(s) P(s+1)\right]\right\|=\sup _{\substack{w \in D(\mathbb{N}(0), X) \\
\|w\|_{\infty} \leq 1}} \sup _{n \geq 0}\left\|\frac{x}{P(n)}+(S w)(n)\right\| \\
=\sup _{\substack{w \in D(\mathbb{N}(0), X) \\
\|w\|_{\infty} \leq 1}}\left\|\frac{x}{P}+(S w)\right\|_{\infty} \geq \sup _{\substack{w \in D(\mathbb{N}(0), X) \\
\|w\|_{\infty} \leq 1}}\|(S w)\|_{\infty}=\|S\| .
\end{gathered}
$$

which holds for all $x \in X$. Ultimately,

$$
\inf _{x \in X} \sup _{\substack{w \in D(\mathbb{N}(0), X) \\\|w\|_{\infty} \leq 1}} \sup _{n \geq 0}\left\|\frac{1}{P(n)}\left[x+\sum_{s=0}^{n-1} w(s) P(s+1)\right]\right\| \geq\|S\|=\beta_{p} .
$$

This completes the proof of the theorem.

EXAMPLE 2.7 Consider

$$
\left(T_{p} u\right)(n)=\triangle u(n)-\left(1+(-1)^{n}\right) u(n)
$$

such that $1+p(n)=2+(-1)^{n}$ and $P(n)=\left(\prod_{i=0}^{n-1}\left(2+(-1)^{i}\right)\right)^{-1}$. Indeed,

$$
\begin{aligned}
\frac{1}{P(n)} \sum_{m=n}^{\infty} P(m+1) & =\frac{1}{\left(2+(-1)^{n}\right)}\left[1+\frac{1}{\left(2+(-1)^{n}\right)}+\frac{1}{\left(2+(-1)^{n}\right)\left(2-(-1)^{n}\right)}+\ldots\right] \\
& \leq 2\left[1+\frac{1}{1.3}+\frac{1}{1^{2} .3^{2}}+\ldots\right]=3
\end{aligned}
$$

implies that $\alpha_{p} \leq 3$. Hence by Theorem 2.1, $T_{p}$ has the Hyers-Ulam stability with HUS constant $\alpha_{p} \leq 3$.

\section{References}

[1] C. Alsina, R. Ger; On some inequalities and stability results related to the exponential function, J. Inequ. Appl., 2(1998), 373-380. 
[2] T. Aoki; On the stability of the linear transformation in Banach spaces, J. Math. Soc. Japan, 2(1950), 64-66.

[3] D. G. Bourgin; Classes of transformations and bordering transformations, Bull. Amer. Math. Soc., 57(1951), 223-237.

[4] J. Brzdek, D. Popa, B. Xu; Remarks on stability of linear recurrence of hogher order, Appl. Math. Lett., 23(2010), 1459-1463.

[5] D. H. Hyers; On the stability of the linear functional equations, Proc. Natl. Acad. Sci., 27(1941), 222-224.

[6] D. H. Hyers, G. Isac, Th. M. Rassias; Stability of Functional Equations in Several Variables, Birkhauser, Basel, 1998.

[7] K. W. Jun, Y. H. Lee; A generalization of the Hyers-Ulam-Rassias stability of the Jenson's equation, J. Math. Anal. Appl., 238(1999), 305-315.

[8] S. M. Jung; Hyers-Ulam stability of linear differential equations of first order, Appl. Math. Lett., 17(2004), 1135-1140.

[9] S. M. Jung; Hyers-Ulam stability of linear differential equations of first order(III), J. Math. Anal. Appl., 311(2005), 139-146.

[10] S. M. Jung; Hyers-Ulam stability of linear differential equations of first order(II), Appl. Math. Lett., 19(2006), 854-858.

[11] S. M. Jung; Hyers-Ulam-Rassioas Stability of Functional Equations in Nonlinear Analysis, Springer, 2011.

[12] S. M. Jung; Hyers -Ulam stability of the first order matrix difference equations, Adv. Differ. Equs., 2015(2015), 1-13.

[13] Y. Li, L. Hua; Hyers-Ulam stability of a polynomial equation, Banach J. Math. Anal., 3(2009), 86-90.

[14] T. Miura, S. E. Takahasi, H.Choda; On the Hyers-Ulam stability of real continuous function valued differentiable map, Tokyo J. Math., (2001), 467-476.

[15] T. Miura; On the Hyers -Ulam stability of a differentiable map, Sci. Math. Japan, 55(2002), 17-24.

[16] T. Miura, S. M. Jung, S. E. Takahasi; Hyers-Ulam stability of the banach space valued linear differential equations $y^{\prime}=\lambda y$, Korean Math. Soc., 41(2004), 995-1005.

[17] T. Miura, H. Oka, S. E. Takahasi, N. Niwa; Hyers-Ulam stability of the first order linear differential equations for Banach space valued holomorphic mappings, J. Math. Ineq., 1(2007), 377-385. 
[18] M. Obloza; Hyers stability of the linear differential equation, Rocznik Nauk-Dydakt, Proce. Mat., 13(1993), 259-270.

[19] D. Popa; Hyers-Ulam-Rassias stability of a linear recurrence, J. Math. Anal. Appl., 309(2005), 591-597.

[20] Th. M. Rassias; On the stability of linear mapping in Banach spaces, Proc. Amer. Math. Soc., 72(1978), 297-300.

[21] Th. M. Rassias; On the stability of functional equations and a problem of Ulam, Acta Appl. Math., 62(2000), 23-30.

[22] S. E. Takahasi, H. Takagi, T. Miura, S.Miyajima; The Hyers-Ulam stability constants of first order linear differential operators, J. Math. Anal. Appl., 296(2004), 403-409.

[23] A. K. Tripathy, A. Satapathy; Hyers-Ulam stability of fourth order Euler's differential equations, J. Comp. Sci. Appl. Math., 1(2015), 49-58.

[24] A. K. Tripathy; Hyers-Ulam stability of second order linear difference equations, Int. J. Diff. Equs. Appl., 1(2017), 53-65.

[25] S. M. Ulam; Problems in Modern Mathematics, Chapter VI, Wiley, New York(1964). 